\title{
El Compromiso Ocular en la Enfermedad de Chagas Congénita
}

\author{
Dr. Antonio Atias M. 1; Dra. Margarita Morales N.2; \\ Dra. Patricia Mữoz C. del V.1; Dra. Marcela Barría C. 3 \\ Ocular lnyolvement in Congenital Chagas' Disease
}

Ocular involvement was demonstrated in 9 out of 11 cases of Congenital Chagas' Disease. The foltowing changes ocurred at the posterior pole of the eye: Papilar edema, small retinal hemorrhages, chorioretinitis of variable aspects, macular involvement and preretinal vitreous opacities. The lesions appeared isolated or in combination. Postmortem examination in one child revealed a lymphoplasmocytic infiltration of the choroid and the retina. The type of the lesions observed was not pathogonomonic and apparently there was no correlation either between the severity of the clinical and the ocular picture, or between the time of evolution and the severity of the lesions. Improvement was observed in children submitted to primaquine or nifurtimox treatment. The relatively high incidence of ocular involvement observed in these cases, seems to indicate that eye fundus examination should be performed routinely in all children in whom congenital Chagas' Disease is suspected or evident.

(Key words: Chagas' Disease. Congenital. Ocular Involvement. Nifurtimox. Transplacental Transmission).

En la enfermedad de Chagas congénita, el Trypanosoma cruzi provoca una infección generalizada del feto, con abundantes formas san. guíneas del parásito (tripomastigotos) que diseminan la infección por todo el organismo, $y$ gran cantidad de pseudoquistes en los tejidos (amas. tigotos), los que se multiplican en prácticamente todos los órganos, sobre todo en el sistema retículoendotelial y en la musculatura esquelétical.

Los hallazgos más caracteristicos de la forma congénita de la infección son: bajo peso de nacimiento (prematuro o pequeño para la edad gestacional), hepatomegalia, esplenomegalia y compromiso variable del sistema nervioso central y miocardjo ${ }^{2}$. Estos síntomas son indiferenciables de los provocados por otros agentes infecciosos que puedan afectar al recién nacido in utero (TORCH). Sin embargo, en nuestra experiencia, algunos niños infectados pueden nacer aparentemente sanos y desarrollar después de meses, alteraciones meningoencefálicas o aumento de volumen de higado y del bazo.

1. Unidad de Parasitología, División Cs. Médicas Occidente, Facultad de Medicina, Universidad de Chile.

2. Servicio de Oftalmología Infantil, Hospital San Juan de Dios, Santiago.

3. Departamento y Servicio de Pediatría, Hospital San Juan de Dios, Santiago.

(*) Financiado en parte por grant del Tropical Disease Research / WHO T 16/181/74 c. kd. 820599.
Entre 1961 y 1962 comunicamos los primeros casos del compromiso ocular en ta enfermedad de Chagas congénita ${ }^{3-4}$ y en 1963 , describimos los hallazgos histopatológicos oculares en un niño fallecido por esta afección parasitaria ${ }^{5}$. Posteriormente, hemos estudiado el compromiso ocular en los casos de enfermedad de Chagas congénita diagnosticados por nosotros, $y$ fueron dados a conocer previamente como una comunicación preliminar ${ }^{6}$.

\section{MATERIAL Y METODO}

En el Hospital San Juan de Dios, Santiago de Chile, zona de baja endemia chagásica, diagnosticamos entre uno y dos casos anuales de enfermedad de Chagas congénita sintomática. El diagnóstico se sospecha cuando existen antecedentes epidemiológicos maternos y maniffestaciones clínicas en el niño y se confirma cuando resultan positivas la reacción de inmunofluorescencia indirecta, la reacción de hemaglutinación indirecta o ambas y se aisla $T$. cruzi en los exámenes directos de sangre, por gota gruesa, $y$ xenodiagnóstico.

Para examinar el fondo de ojos de estos niños, se indujo midriasis instilando solución de Homa. tropina al 2\%, una gota en cada ojo, cada 10 minutos, 3 veces. El examen fue hecho por uno de nosotros (MM) mediante of talmoscopía directa.

En esta revisión, se analizaron 11 casos de 
enfermedad de Chagas congénita, entre los que están incluidos tres publicados previamente (Casos 7, 8 y 9).

\section{RESULTADOS}

De un total de 11 casos de enfermedad de Chagas congénita comprobada, en los que se efectuó estudio ocular, 9 tenían lesiones del ojo. Las madres de estos niños provenian de zonas de baja endemia, eran asintomáticas y dos tenian antecedentes de haber recibido transfusiones san- guineas.

En la Tabla 1 se muestran los hallazgos clinicos fundamentales en los 9 pacientes con com. promiso ocular: En 5 (Casos 1, 2, 3, 4 y 5 ), el diagnóstico de la enfermedad se efectuó en el periodo de recién nacido; todos fueron de pretérmino, su peso de nacimiento osciló entre 1.300 y 2.650 gramos; tenían hepatomegalia, esplenomegalia de grado variable y en 2 existia, además, compromiso meningoencefálico (Casos 3 y 5).

Tabla I

Cornpromiso Ocular en la Enfermedad de Chagas Congenita. Cuadro Clínico, Evolución y Tratamiento en 9 Casos

\begin{tabular}{|c|c|c|c|c|c|c|c|c|}
\hline . & $\begin{array}{l}\text { Edadl del } \\
\text { Diagnóstico }\end{array}$ & $\begin{array}{c}\text { Poso } \\
\text { Vacintuiento }\end{array}$ & $\begin{array}{l}\text { Hepa toesple- } \\
\text { nomegalia }\end{array}$ & $\begin{array}{l}\text { Compromiso } \\
\text { S.N.C. }\end{array}$ & $\begin{array}{l}\text { Alteraciones } \\
\text { Cardjacas. }\end{array}$ & $\begin{array}{l}\text { Otras Ait. } \\
\text { Clínicas. }\end{array}$ & Evolución & Tratamiento \\
\hline 1) C.V. & $14 \mathrm{ds}$ & 1.300 & + & & & Púrрuта & Fallece & \\
\hline 2) V.M. & $10 \mathrm{ds}$. & 1.995 & + & & + & Jcterícia & Regular & Nifuttitnox \\
\hline 3) R.R. & $20 \mathrm{ds}$ & 1.550 & + & + & + & & Regular & Njrurtimox \\
\hline 4) R.H. & $20 \mathrm{ds}$ & 1.800 & + & & & & Buena & Primaquina \\
\hline 5) J.R. & $2 \mathrm{ds}$. & 2.650 & + & + & + & & Fallece & \\
\hline 6) A.H. & 3 mesies & 3.550 & + & & + & Pürpura & Buena & Niturtionox \\
\hline 7) L.E. & $1 \mathrm{m.} 10 \mathrm{ds}$ & 2.180 & + & + & + & & Buena & Nifurtionox \\
\hline 8) M.C. & 1 ano $4 \mathrm{~ms}$. & 3.200 & & + & + & $\begin{array}{l}\text { Estrabismo } \\
\text { Convergente }\end{array}$ & Buenta & Nifurtimox \\
\hline 9) V.A. & 11 meses & 3.000 & & & & Mcgaesófago & Buena & Primaguina \\
\hline
\end{tabular}

En los otros 4 niños (Casos $6,7,8$ y 9) el diagnóstico de enfermedad de Chagas se hizo durante la lactancia; en ellos descartamos, hasta donde fue posible, el antecedente de contacto con triatomideos; y ninguno recibió transfusiones sanguíneas. Por estas razones, los consideramos congénitamente infectados. De éstos, sólo uno (Caso 7) nació antes del término con 2.180 gramos de peso: los motivos de su hospitalización fueron crisis convulsivas, irritación meningea, hepatomegalia de $3 \mathrm{~cm}$. y esplenomegalia de $1 \mathrm{~cm}$.

En el Caso 6 el diagnóstico se hizo a los 3 meses de edad. Después de un periodo de recién nacido normal, presentó gran compromiso del estado general, hepatomegalia de $5 \mathrm{~cm}$., esplenomegalia de $6 \mathrm{~cm}$. y pútpura con petequias en la cabeza y el tronco. El caso 8 corresponde a una nifra con retraso psicomotor, estrabismo convergente e hipoacusia, y en quién se aislo $\mathrm{T}$. cruzi a la edad de 1 año y 4 meses. E1 caso 9 consultó por disfagia y vómitos debidos a megaesófago; el diagnóstico de la enfermedad de Chagas se hizo a los 11 meses de edad.

En 2 niños se observaron alteraciones importantes en el electrocardiograma: En el Caso 6, signos de crecimiento de ventriculo derecho, bloqueo incompleto de rama derecha del haz de Hiss y trastornos de la repolarización; en el Caso 5, signos de reacción auricular y ventricular derechos. Los demás pacientes sólo presentaron taquicardia sinusal.

Las lesiones oculares se pesquisaron mediante el examen de fondo de ojos en 8 casos (Tabla 2). El caso restante (Caso 5), falleció precozmente, pero la necropsia reveló infilltración linfoplasmo. citaria de corojdes y retina, sin que se pudiera encontrar el parásito en esos tejidos. En los 8 casos en que se hizo fondoscopia, se encontraron lesiones del polo posterior del ojo, con compromiso de papila, coroides, retina y humor vítreo. 
Tabla 2.

Compromiso Ocular en la Enfermedad de Chagas Congénita

Exámen de Fondo de Ojos en 9 Casos

\begin{tabular}{|c|c|c|c|}
\hline Caso & Ex. de F. de O. Inicial & Utimo Control (Post - Tratamiento) & Tiempo de Eyolución \\
\hline I.- C.V. & $\begin{array}{l}\text { OD: Foco de cotiorretinitis irregularmente } \\
\text { pigmentado, subpapilar, de } 2 \text { D.P. }\end{array}$ & Falleció & - \\
\hline 2. V.M. & $\begin{array}{l}\text { O1: Dos pequeñas hemorragias en rama } \\
\text { temporal superior. }\end{array}$ & Normal & 2 meses \\
\hline 3.- R.R. & ODI: Edema papilar discreto & $\begin{array}{l}\text { Leve paldez sector temporal de ambas } \\
\text { papilas. Mácula con finos depósitos } \\
\text { dorados, sín estructura foveolar. }\end{array}$ & 9 meses \\
\hline 4.- N.H. & $\begin{array}{l}\text { OD: Papila de bordes discretamente bortosos. } \\
\text { Edema retinal discreto. Hacia nasal } 4 \mathrm{DP} \text {, } \\
\text { solevaniamiento de } 2 \mathrm{DP} \text { amarillo grisaceo. }\end{array}$ & No se controló & - \\
\hline S. J.R. & $\begin{array}{l}\text { Anat, Patológica ODI: Infiltración } \\
\text { linfoplasmocitaria de coroides y retina. }\end{array}$ & F'allecjó & - \\
\hline 6.- A.H. & $\begin{array}{l}\text { DDI: Papilas levemente pálidas, miculas con } \\
\text { depósitos dorados sin brillo foveolar, } \\
\text { hemorragia pequeña en CTS. Aspecto } \\
\text { "vitrificado" dr la retina en el polo posterior. }\end{array}$ & $\begin{array}{l}\text { Papilas levernente pálidas, máculas sin } \\
\text { brillo foveolar. }\end{array}$ & 1 año 6 ineses \\
\hline $7 . \mathrm{LE}$ & $\begin{array}{l}\text { ODI: Papilas discretamente pálida5. Mäculas } \\
\text { con depósitos dorados, sín brillo. Entre } \\
\text { papila y mácula, aspecto "vittificađo" de ta } \\
\text { retina. }\end{array}$ & Idem & 1 año 5 meses \\
\hline 8. M.C. & $\begin{array}{l}\text { OD: Papila de bordes hiperémicos } \\
\text { Ol: Sobre Ja papila hay dos velos vitreos } \\
\text { tenues. Retina grisácea. }\end{array}$ & Normal & 2 años \\
\hline $9 \cdot V \cdot A$ & $\begin{array}{l}\text { OD: Ligera opacidad vitrea pre-macular } \\
\text { ODl: Retina en "sal y pimienta". }\end{array}$ & $\begin{array}{l}\text { ODI: Dispersión pigmentaria macular } \\
\text { Finas pigmentaciones doradas comprometen } \\
\text { la retina cercana a las máculas. }\end{array}$ & 18 aกิดs \\
\hline
\end{tabular}

En 5 pacientes (Casos 3, 4, 6, 7 y 8 ) había lesiones de la papila; en 3 edema leve $y$ en 2 palidez papilar discreta, probablemente postedema (Fig. 1). En 2 niños (Casos 6 y 7) se observó en la mácula dispersión pigmentaria y ausencia del brillo foveolar (Fig. 2). En la retina se encontraron pequeñas hemorragias (Casos 2 y 6) y aspecto "vitrificado" (Casos 6 y 7) o en "sal y pimienta" (Caso 9) (Fig. 3); en un paciente (Caso 4), existian edema retinal discreto y pequeños solevantamientos amarillo grisáceos de la retina. Un solo enfermo (Caso 1), con peso de nacimiento de 1.300 gramos, he patoesplenomegalia, púrpura, y que falleció a los pocos días de nacido, presentó un gran foco de corioretinitis subpapilar, de 2 diámetros papilares, bien delimitado e irregularmente pigmentado, semejante al descrito en la toxoplasmosis ocular antes que se haga necrotizante. Dos niños presentaron tenues velos vítreos prepapilar (Caso 8) (Fig. 4) y premacular (Caso 9).

El tratamiento de la infección tripanosómica se efectuó en 5 niños con Nifurtimox en dosis de $15 \mathrm{mg}$. por $\mathrm{Kg}$. de peso por día, durante 60 días. Los Casos 4 y 9 recibieron Difosfato de primaquina, $1 \mathrm{mg}$. por $\mathrm{Kg}$. de peso por dia, durante 14 días. Dos enfermos fallecieron poco después de haberse hecho el diagnóstico y antes de iniciar el tratamie nto específico.

Se is de los ocho niños tienen control fondoscópico post-tratamiento. En 2 la recuperación fue total (Casos 2 y 8). Tres presentaron leve palidez papilar y mácula sin brillo foveolar (Casos 3,6 y 7 ). El caso 9 que en la infancia tenia opacidad vítrea y retina en "sal y pimienta", presenta, 18 años después, sólo dispersión pigmentarja de las máculas y la retina que las rodea, siendo su visión, normal. 


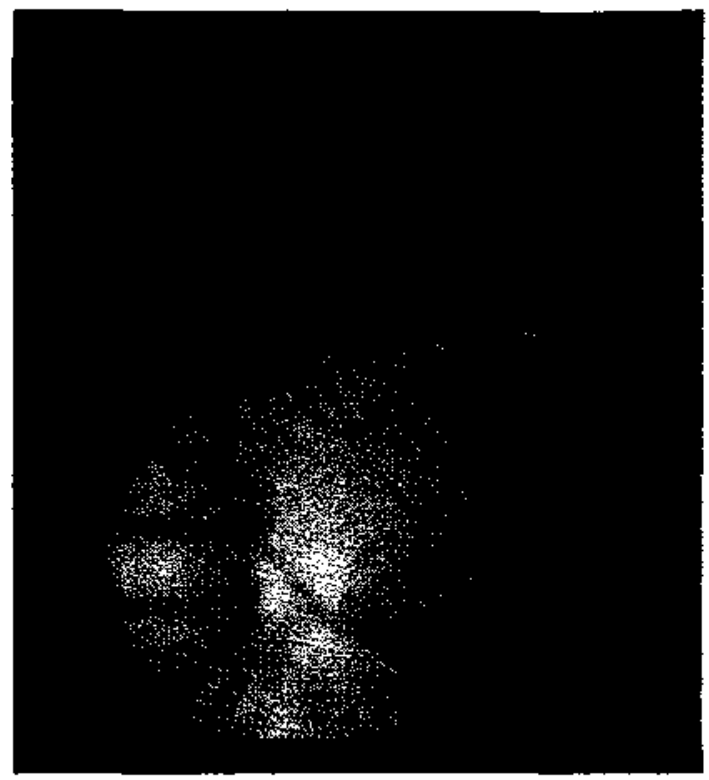

Figura 1.

Edema papilar leve.
Figura 3.

Retina en "sal y pimienta"

(Corioretinitis leve).

\section{DISCUSION}

La enfermedad de Chagas congénita no es rara en nuestro medio y se la debiera investigar en recién nacidos provenientes de zona endémica con síndrome de infección transplacentaria, $y$ en lactantes menores con sintomas atribuibles al mismo síndrome.

Llama la atención la alta frecuencia $(9 / 11)$ del

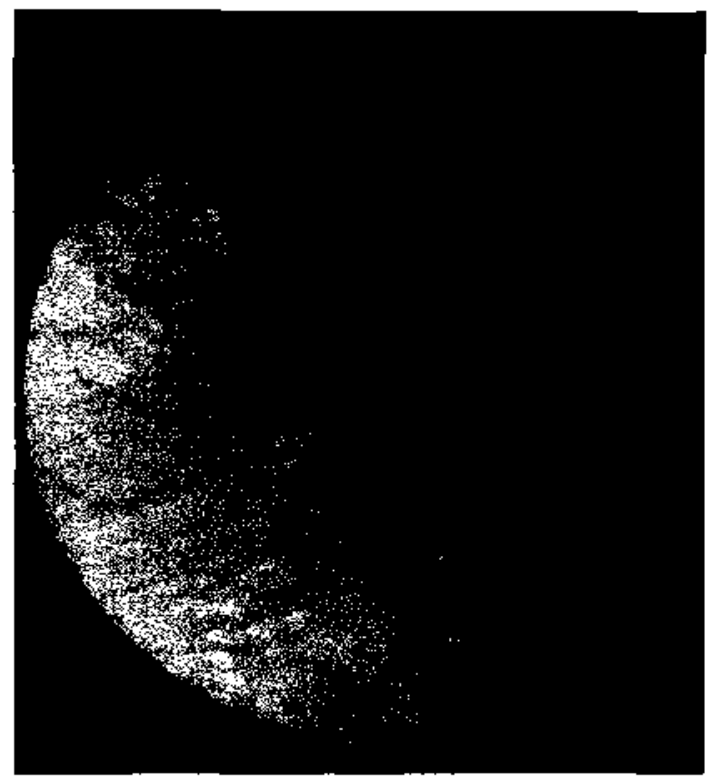

Figura 2.

Mácula con dispersión pigmentaria y ausencia del brillo foveolar.

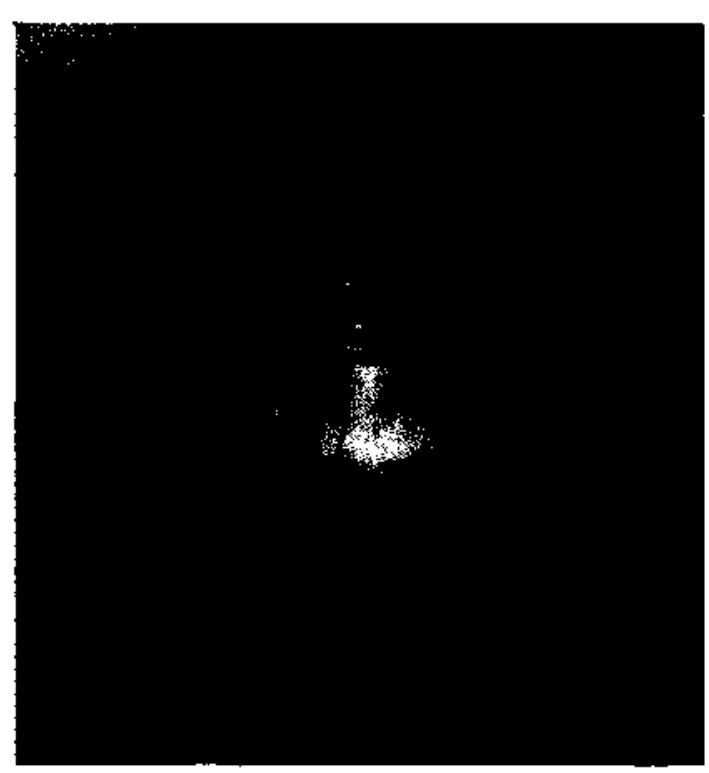

Figura 4.

V'los prepapilares (Compromiso del vítreo).

compromiso del polo posterior del ojo en los pacientes reseñados. En los Casos 1 a 5 , e] diagnóstico de enfermedad de Chagas congénita no merece dudas, pues se hizo en el período de recién nacido antes de dejar el hospital. Los 4 restantes, han sido considerados infectados con. génitamente, después de descartar la presencia de triatomas en los domicilios y el suministro de transfusiones sanguíneas; sin embargo, no po- 


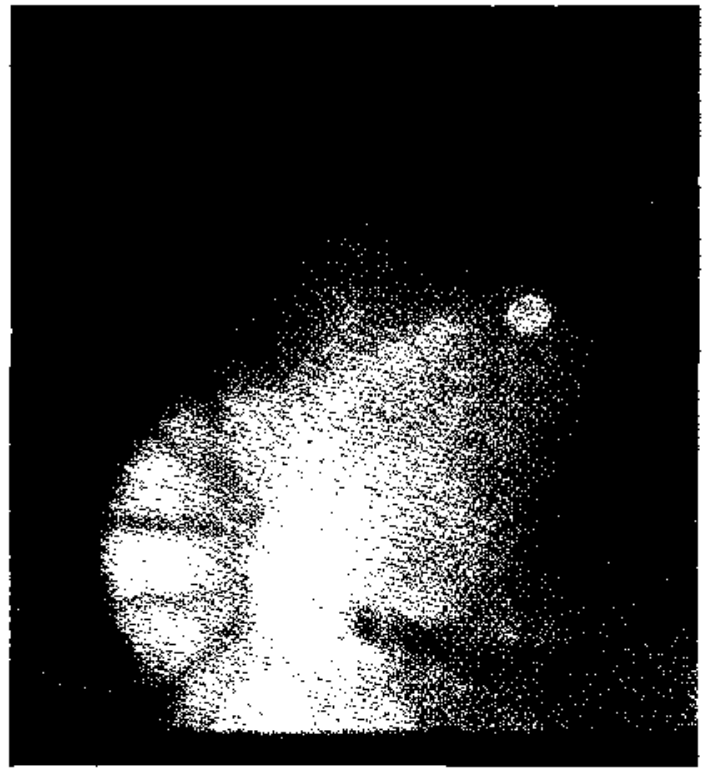

Figura 1.

Fona papilar love.

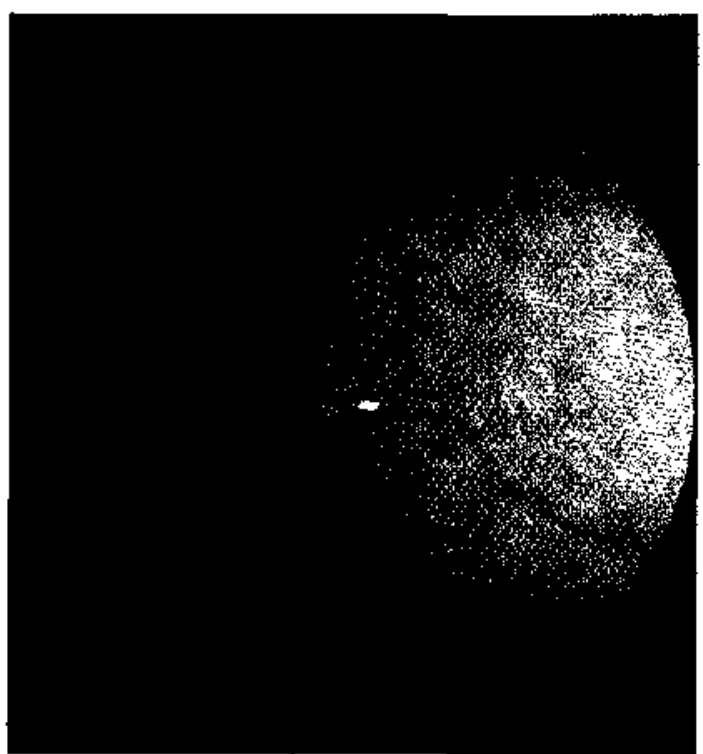

Figura 3.

Retina en "sal y pimienta" (Corioretinitis leve).

\section{DISCUSION}

La enfermedad de Chagas congénita no es rara en nuestro medio y se la debiera investigar en recién nacidos provenientes de zona endémica con sindrome de infección transplacentaria, y en lactantes menores con sintomas atribuibles al mismo sindrome.

Llama la atención la alta frecuencia $(0 / 1) \mathrm{del}$

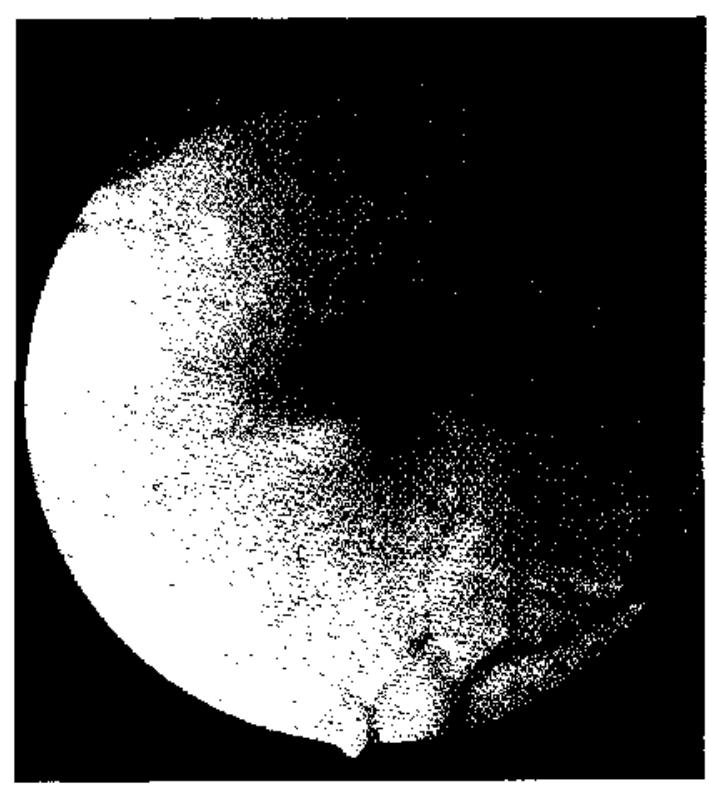

ligura 2.

Mácula con dispersión pigmentaria y ausencia del brillo tovecolar.

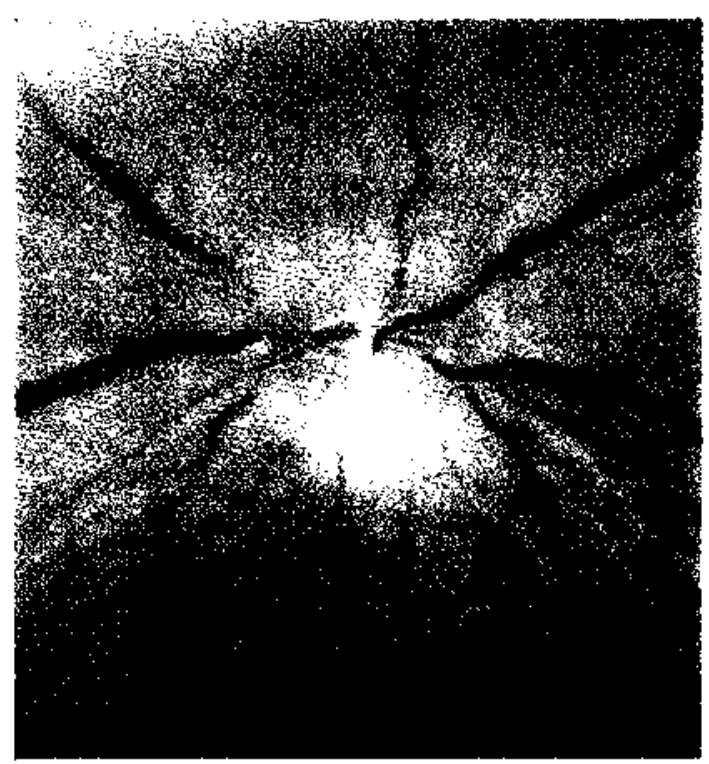

Figura 4.

Velos prepapilares (Compromiso del vilreo).

compromiso del polo posterior del ujo en los pacientes reseñados. En los Casos 1 a 5 , el diagnóstico de enfermedad de Chagas congénita no merece dudas, pues se hizo en el periodo de recién nacido antes de dejar el hospital. Los 4 restantes, han sido considerados infectados congénitamente, después de descartar la presencia de triatomas en los domicilios y el suministro de transfusiones sanguineas; sin embargo, no po- 